\title{
Morfometria do forame mandibular de Cerdocyon thous (Linnaeus, 1766) (cachorro-do-mato)
}

\author{
Paulo de Souza Junior ${ }^{1 *}$ \\ Rodrigo Jiddu Ferreira Pinto \\ Adriano Biase de Freitas ${ }^{2}$ \\ Natan da Cruz de Carvalho ${ }^{1}$ \\ ${ }^{1}$ Laboratório de Anatomia Animal, Universidade Federal do Pampa \\ BR-472, km 592, Caixa Postal 118, CEP 97500-970, Uruguaiana - RS, Brasil \\ ${ }^{2}$ Laboratório de Anatomia Animal, Universidade Castelo Branco, Rio de Janeiro - RJ, Brasil \\ * Autor para correspondência \\ paulosouzajr@terra.com.br
}

Submetido em 16/09/2012

Aceito para publicação em 19/01/2013

\section{Resumo}

O bloqueio anestésico do nervo alveolar inferior é a técnica mais indicada para procedimentos cirúrgicos nos dentes inferiores e mandíbula de animais e seres humanos. Sua execução depende do conhecimento da topografia do forame mandibular da espécie abordada. Este estudo realizou mensurações de 6 pontos de referência anatômica em 20 hemimandíbulas de 10 cadáveres adultos ( 3 machos e 7 fêmeas) de cachorro-do-mato, para estabelecer a topografia do forame mandibular e, assim, oferecer subsídios para o bloqueio do nervo alveolar inferior nessa espécie. Houve uma variação significativa $(\mathrm{p}=0,05)$ no posicionamento do forame em relação à borda ventral da mandíbula entre indivíduos de sexos diferentes. Não houve variação entre antímeros. Os resultados indicam que $20,4 \mathrm{~mm}$ da agulha devem ser introduzidos obliquamente a partir do processo angular e 12,1 mm (fêmeas) ou 13,6 mm (machos) perpendicularmente a partir da borda ventral da mandíbula, para que o anestésico seja administrado próximo ao forame mandibular e, desse modo, tenha maior chance de proporcionar o bloqueio pretendido. Foram observadas diferenças entre cães domésticos e cachorro-do-mato quanto à forma e topografia de pontos de referência empregados em técnicas de bloqueio, especialmente na localização mais rostral do forame mandibular no Cerdocyon thous.

Palavras-chave: Anatomia animal; Cachorro-do-mato; Canídeos silvestres; Nervo alveolar inferior

\section{Abstract}

Morphometrics of the mandibular foramen of Cerdocyon thous (Linnaeus, 1766) (crab-eating-fox). Anesthetic block of the inferior alveolar nerve is the most indicated technique for surgical procedures in the lower teeth and jaw of animals and human beings. Its execution depends on knowing the topography of the mandibular foramen in the species addressed. This study performed measurements in 6 anatomical reference sites in 20 hemimandibles of 10 adult cadavers ( 3 males and 7 females) of crab-eating-fox, in order to establish the topography of the mandibular foramen and, thus, provide support for blocking the inferior alveolar nerve in this species. There was a significant variation $(p=0.05)$ in the foramen position with regard to the ventral border of the jaw among individuals of both sexes. There was no significant variation among antimers. The 
results indicate that $20.4 \mathrm{~mm}$ of the needle should be obliquely inserted from the angular process and $12.1 \mathrm{~mm}$ (females) or $13.6 \mathrm{~mm}$ (males) of it should be perpendicularly inserted from the ventral border of the jaw, so that the anesthetic is administered close to the mandibular foramen and, thus, have a greater chance of providing the desired block. Differences were observed between domestic dogs and crab-eating-fox with regard to the shape and topography of reference sites used in blocking techniques, especially in the more rostral location of the mandibular foramen in Cerdocyon thous.

Key words: Animal anatomy; Crab-eating-fox; Inferior alveolar nerve; Wild canids

\section{Introdução}

O Cerdocyon thous, conhecido por cachorro-domato ou graxaim-do-mato, é um canídeo silvestre de ocorrência em todo território brasileiro (a exceção do Acre e Amazonas), bem como na Colômbia, Venezuela, Uruguai, Paraguai e nordeste Argentino (BERTA, 1982; MACHADO; HINGST-ZAHER, 2009). Os adultos possuem massa corporal entre quatro e $11 \mathrm{~kg}$, a cabeça e tronco medem entre 60 e $70 \mathrm{~cm}$ e a cauda cerca de 30 $\mathrm{cm}$. Com hábitos noturnos e crepusculares, é uma espécie onívora, generalista e oportunista que possui aparelho mastigatório adaptado para uma dieta à base de pequenos vertebrados, frutos e invertebrados (LANGGUTH, 1975; BERTA, 1982). A espécie é comumente exibida em jardins zoológicos de todo o mundo e, apesar de atualmente não ser ameaçada de extinção, muitas populações sofrem os impactos por atropelamentos nas rodovias brasileiras (CHEIDA et al., 2011).

Uma das causas mais comuns de atendimentos veterinários em carnívoros silvestres destina-se ao tratamento das afecções dentárias. Os traumas com exposição da polpa dentária e consequente fístula mandibular são muito frequentes e podem decorrer de acidentes em grades e concreto dos recintos, brigas com outros animais ou ainda por efeito de ação deletéria de humanos (corte de dentes). Procedimentos que visam preservar a dentição e a oclusão mantêm a integridade do aparelho mastigatório e asseguram a ingestão adequada de alimentos (PACHALY; GIOSO, 2001).

O forame mandibular dos carnívoros fica localizado na face medial do ramo da mandíbula, ventralmente ao ponto de inserção do músculo temporal, e corresponde à abertura caudal do canal mandibular. Este canal se abre rostralmente nos forames mentonianos e permite a passagem da artéria, veia e nervo alveolares inferiores. $\mathrm{O}$ nervo alveolar inferior é um ramo do nervo mandibular que surge na face lateral do músculo pterigoide medial, origina o nervo miloioideo e penetra no canal mandibular pelo forame mandibular. Ao longo do seu trajeto dentro do canal, forma os ramos alveolares inferiores (caudal, médio e rostral) e o plexo dentário inferior com função sensorial para os dentes inferiores e gengiva. Próximo à extremidade rostral da mandíbula emerge pelo forame mentoniano originando os nervos mentonianos que fornecem ramos sensoriais para o lábio inferior e região intermandibular rostral (DELLMANN; MCCLURE, 1986; EVANS; KITCHELL, 1993; DYCE et al., 2004; EVANS; LAHUNTA, 2010; KÖNIG et al., 2011; INTERNATIONAL COMMITTEE ON VETERINARY GROSS ANATOMICAL NOMENCLATURE, 2012).

O bloqueio regional do nervo alveolar inferior é eficiente em anestesiar todos os dentes inferiores e tecidos moles ipsilaterais e pode ser feito por técnica intra-oral ou extra-oral em cães domésticos (GROSS et al., 1997; HOLMSTROM et al., 2004; HALE, 2007; EGGER; LOVE, 2009). Na técnica intra-oral, tenta-se palpar o forame mandibular na face medial da mandíbula a dois terços da distância desde o terceiro molar ao processo angular e aplica-se o anestésico neste ponto (BECKMAN; LEGENDRE, 2002; HOLMSTROM et al., 2004). Na técnica extra-oral (técnica de Akinosi), palpa-se uma concavidade na região caudal da margem ventral da mandíbula e, a partir deste ponto, avança-se a agulha dorsalmente junto à face medial da mandíbula (HOLMSTROM et al., 2004; EGGER; LOVE, 2009). Em alguns cães consegue-se palpar o forame mandibular na face medial do ramo mandibular (HOLMSTROM et al., 2004). Beckman e Legendre (2002) e Hale (2007) indicam que a agulha deva ser avançada dorsalmente desde a borda ventral até que atinja metade da altura do corpo da mandíbula. 
Esta técnica de bloqueio apresenta vantagens que a torna justificável como, por exemplo, permitir analgesia durante várias horas após o procedimento cirúrgico, melhorar o efeito de narcóticos sistêmicos utilizados no pré-operatório, diminuir o plano de anestesia geral ou mesmo dispensar a prescrição de analgésicos no pósoperatório, além da facilidade de execução e baixo custo (BECKMAN; LEGENDRE, 2002; HOLMSTROM et al., 2004; HALE, 2007). Os benefícios do bloqueio regional podem ser ainda mais desejáveis em medicina de canídeos silvestres pela dificuldade em administrar medicamentos no pós-operatório, alta frequência de fraturas, incluindo as mandibulares e dentárias, além de frequentemente os pacientes se encontrarem debilitados na ocasião do procedimento cirúrgico (PACHALY; GIOSO, 2001; PESSUTTI et al., 2001). Como desvantagens, apontam-se os riscos de bloquear o nervo glossofaríngeo (e o paciente traumatizar a língua quando despertar do procedimento anestésico), trauma iatrogênico ao feixe vasculonervoso (resultando em parestesia temporária) e administração inadvertida de anestésico local intravenosa ou intra-arterial (BECKMAN; LEGENDRE, 2002; HOLMSTROM et al., 2004; HALE, 2007; EGGER; LOVE, 2009).

O bloqueio do nervo alveolar inferior é a mais importante e frequente técnica anestésica regional em seres humanos, ainda que tenha uma porcentagem de falhas alta (15 a 20\%), mesmo quando propriamente administrada (RIZZOLO; MADEIRA, 2006; STRINI et al., 2006). Há vários fatores que interferem no êxito desta modalidade de anestesia, mas os insucessos ocorrem principalmente por imprecisão na localização anatômica do forame mandibular (STRINI et al., 2006; LIMA et al., 2011). As variações individuais na localização do forame em seres humanos são levadas em consideração como uma das causas de insucesso no bloqueio (RIZZOLO; MADEIRA, 2006). Portanto, é razoável supor que a extrapolação de pontos de referências anatômicas utilizados em cães domésticos para o bloqueio anestésico nos canídeos silvestres possa levar a um índice maior de falhas.

O objetivo deste trabalho foi, por meio da morfometria, estabelecer a localização topográfica precisa do forame mandibular no Cerdocyon thous (cachorro-do-mato) a fim de oferecer subsídios para técnicas eficientes de bloqueio anestésico local do nervo alveolar inferior nesta espécie.

\section{Material e Métodos}

Foram utilizadas 20 hemimandíbulas de dez cadáveres adultos (três do sexo masculino e sete do sexo feminino) de Cerdocyon thous encontrados na rodovia BR-116, no trecho entre Duque de Caxias e Além Paraíba (estado do Rio de Janeiro, Brasil). Após a remoção dos tecidos moles da região da cabeça, as mandíbulas foram desarticuladas nas junções têmporomandibular e sínfise mentoniana e removidas dos cadáveres. Em seguida, as mandíbulas foram imersas em solução de álcool etílico hidratado 92,8 $8^{\circ}$ INPM (Coperalcool ${ }^{\mathbb{}}$ ) durante $24 \mathrm{~h}$ e posteriormente deixadas secar a temperatura ambiente por $48 \mathrm{~h}$ para então serem submetidas à maceração por coleópteros da espécie Dermestes maculatus até que não remanescessem tecidos moles. As mandíbulas foram clarificadas através da imersão em solução de peróxido de hidrogênio P.A. a 130 vol. (Brquim ${ }^{\circledR}$ ), com diluição $1: 20$, durante 30 minutos.

Após a clarificação, foram realizadas seis diferentes medições em cada hemimandíbula, por examinador único, utilizando-se um paquímetro de precisão digital (capacidade $0-150 \mathrm{~mm}$, resolução $0,01 \mathrm{~mm}$, exatidão $\pm 0,02 \mathrm{~mm}$, ZAAS Precision, Amatools $\left.{ }^{\circledR}\right)$. Tomando como base o trabalho de Barroso et al. (2009), foram aferidas as seguintes medições (em milímetros) em cada hemimandíbula (Figura 1):

- ELM: distância entre a margem lateral do processo condilar e a raiz do primeiro incisivo inferior.

- ETM: distância entre as bordas ventral e dorsal da mandíbula, tomada entre o quarto pré-molar e o primeiro molar inferiores.

- FMBV: distância da margem rostral do forame mandibular até a borda ventral da mandíbula neste nível.

- FMANG: distância entre a margem rostral do forame mandibular até a extremidade caudal do processo angular.

- FMCOND: distância entre a margem rostral do forame mandibular até a extremidade medial do processo condilar. 
FIGURA 1: Fotografia da face medial da hemimandíbula direita de uma fêmea de Cerdocyon thous, destacando as medidas ELM (1), ETM (2), FMBV (3), FMANG (4), FMCOND (5) e FMCOR (6).

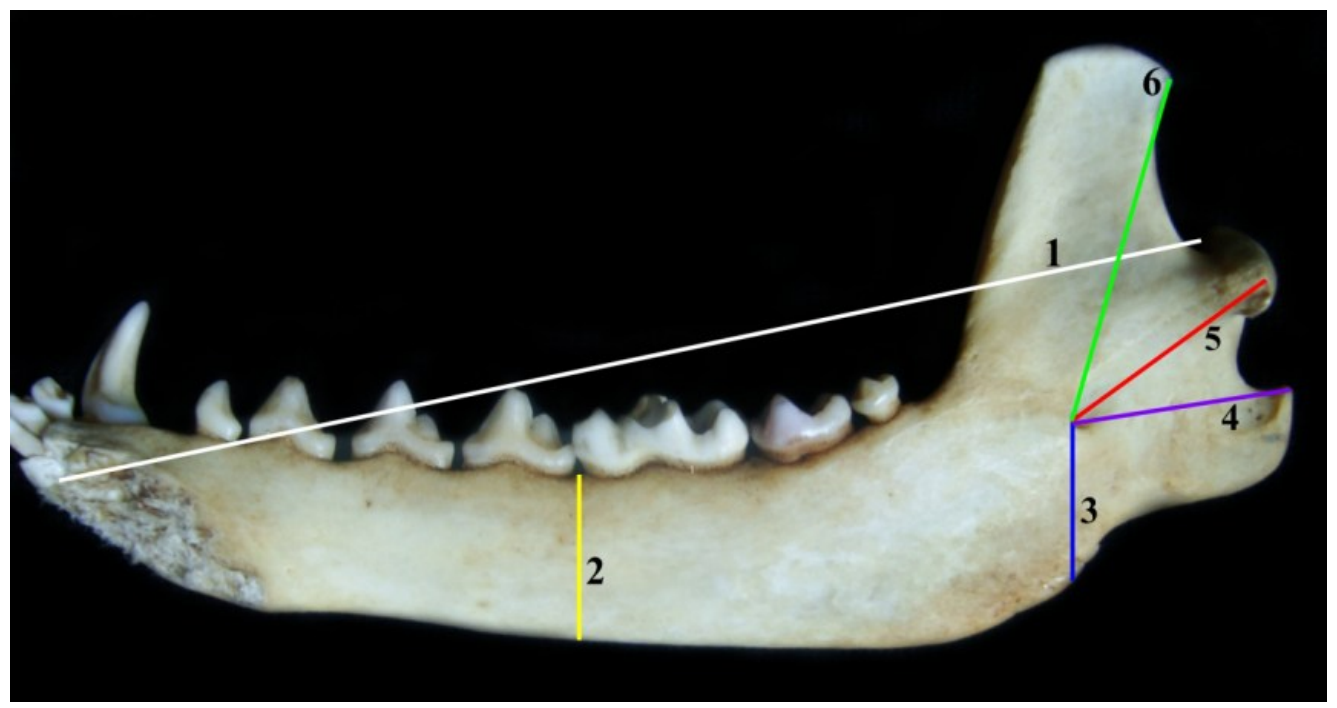

- FMCOR: distância entre a margem rostral do forame mandibular até a extremidade caudal do processo coronoide.

Os resultados destas medições foram submetidos à análise estatística descritiva e ao teste-t de student $(\mathrm{p}=$ 0,05 ) com um intervalo de confiança de 95\% utilizandose o software BioEstat $5.3^{\circledR}$.

Finalmente, observações descritivas e comparativas entre as formas das hemimandíbulas de Canis lupus familiaris (cão doméstico) e Cerdocyon thous (cachorrodo-mato) de portes equivalentes foram registradas.

\section{Resultados}

A presença de um forame mandibular na face medial do ramo da mandíbula, ventralmente ao ponto de inserção do músculo temporal e servindo como abertura caudal para o canal mandibular também foi verificado no Cerdocyon thous (Figura 2) na mesma localização descrita para cães domésticos por Dellmann e Mcclure (1986), Evans e Kitchell (1993), Dyce et al. (2004), Evans e Lahunta (2010), König et al. (2011).

Quando confrontadas as hemimandíbulas de Cerdocyon thous e cães domésticos de porte equivalente, observaram-se nos ossos de cachorros-do-mato processos coronoides mais curtos e com limite caudal menos pontiagudo, incisuras mandibulares menos profundas, processos angulares mais arredondados, amplos e lisos e fossas massetéricas menos profundas (Figura 3). No entanto, a diferença mais relevante foi a disposição rostral do forame mandibular do Cerdocyon thous em relação à concavidade apontada como ponto de referência anatômica para a técnica extra-oral de bloqueio em cães domésticos por Holmstrom et al. (2004) e Egger e Love (2009).

As seis medições propostas foram realizadas em 19 das 20 hemimandíbulas utilizadas. Em apenas uma hemimandíbula esquerda de um cadáver do sexo masculino não foi possível fazer as medições ELM, FMANG, FMCOND e FMCOR devido à fratura com perda de fragmentos. A falta destas medições em apenas uma hemimandíbula não comprometeu o resultado do trabalho, revelando que o aproveitamento de cadáveres recolhidos em rodovias é viável e evita o impacto de metodologias que envolvam capturas para fins científicos.

As Tabelas 1, 2 e 3 demonstram as médias aritméticas e os respectivos desvios padrões das aferições morfométricas (em milímetros) e do valor obtido para p no teste-t de comparação entre as médias.

O eixo longitudinal da mandíbula (ELM) médio foi $99,61 \pm 4,86 \mathrm{~mm}$, não havendo diferença entre as 
FIGURA 2: Fotografias das faces lateral (A) e medial (B) da hemimandíbula direita de fêmea adulta de Cerdocyon thous demonstrando o forame mandibular (FM), processo coronoide (PCOR), processo condilar (COND), processo angular (PANG), forames mentonianos (Fment), fossa massetérica (Fmass) e dentição inferior incisiva (I), pré-molar (PM) e molar (M).
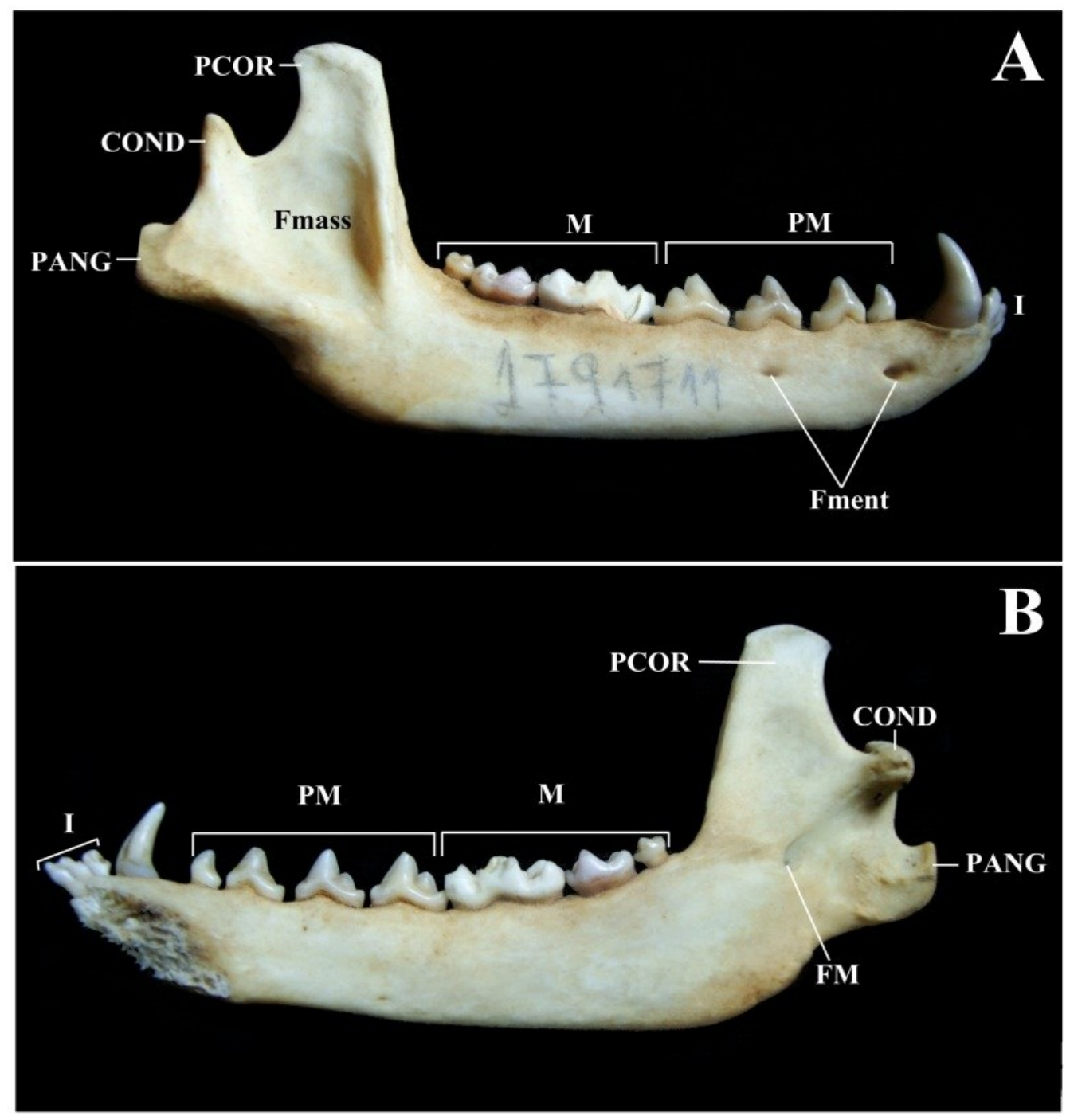

hemimandíbulas esquerdas $(99,25 \pm 5,86 \mathrm{~mm})$ e direitas $(99,93 \pm 4,06 \mathrm{~mm})$. Nas mandíbulas de animais do sexo masculino o ELM foi 103,98 $\pm 5,19 \mathrm{~mm}$, enquanto o de fêmeas foi 98,05 $\pm 3,80 \mathrm{~mm}$. Deste modo, houve diferença estatisticamente significativa ao nível de 5\% quando se comparou o ELM de indivíduos de sexos diferentes $(p=0,0142)$.
O eixo transversal da mandíbula (ETM) médio foi $13,28 \pm 1,18$ mm, não havendo diferenças significativas entre antímeros nem entre sexos. A distância entre o forame mandibular e a borda ventral da mandíbula (FMBV) média foi $12,54 \pm 1,39 \mathrm{~mm}$, não havendo diferença entre as hemimandíbulas esquerdas $(12,20 \pm$ $1,45 \mathrm{~mm})$ e direitas $(12,88 \pm 1,31 \mathrm{~mm})$. 
FIGURA 3: Fotografias comparativas do ramo da mandíbula de fêmeas adultas de porte equivalente Canis lupus familiaris (A: face lateral; C: face medial) e de Cerdocyon thous (B: face lateral; D: face medial). Notar as diferenças de contornos e proporções entre os processos angulares (PANG), coronoides (PCOR) e incisuras mandibulares (IM). No cão doméstico o forame mandibular (FM) situa-se no mesmo nível de maior concavidade da borda ventral da mandíbula, enquanto no Cerdocyon thous o FM encontra-se mais rostral que este nível (seta branca e linha tracejada).

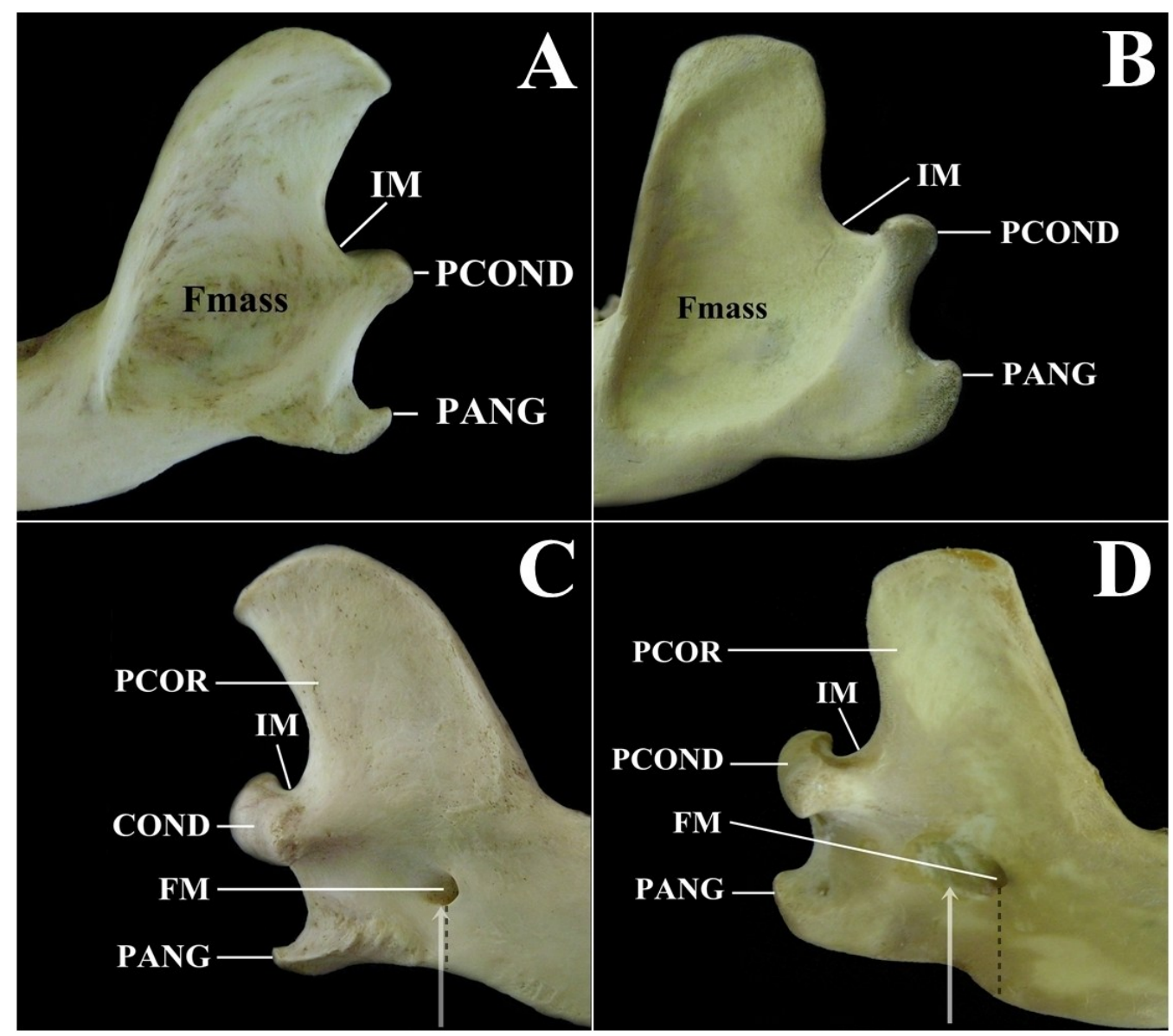

TABELA 1: Resultado em milímetros (média aritmética \pm desvio padrão) das aferições morfométricas realizadas nas hemimandíbulas de Cerdocyon thous $(n=20)$.

\begin{tabular}{cccccc}
\hline ELM & ETM & FMBV & FMANG & FMCOND & FMCOR \\
\hline $99,61 \pm 4,86$ & $13,28 \pm 1,18$ & $12,54 \pm 1,39$ & $20,46 \pm 1,82$ & $19,06 \pm 1,80$ & $27,17 \pm 2,05$ \\
\hline
\end{tabular}

TABELA 2: Resultado em milímetros (média aritmética \pm desvio padrão) das aferições morfométricas realizadas nas hemimandíbulas direitas e esquerdas de Cerdocyon thous e o valor de $p$ obtido no teste t de comparação das médias.

\begin{tabular}{c|cccccc}
\hline & ELM & ETM & FMBV & FMANG & FMCOND & FMCOR \\
\hline Esquerdo & $99,25 \pm 5,86$ & $13,24 \pm 1,23$ & $12,20 \pm 1,45$ & $20,19 \pm 2,35$ & $19,14 \pm 1,99$ & $27,42 \pm 2,11$ \\
Direito & $99,93 \pm 4,06$ & $13,32 \pm 1,21$ & $12,88 \pm 1,31$ & $20,71 \pm 1,23$ & $18,99 \pm 1,72$ & $26,96 \pm 2,09$ \\
p-valor & 0,7732 & 0,8964 & 0,2888 & 0,5418 & 0,8713 & 0,6414 \\
\hline
\end{tabular}


TABELA 3: Resultado em milímetros (média aritmética \pm desvio padrão) das aferições morfométricas realizadas nas

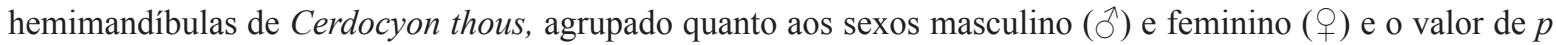
no teste $\mathrm{t}$ de comparação das médias.

\begin{tabular}{c|cccccc}
\hline & ELM & ETM & FMBV & FMANG & FMCOND & FMCOR \\
\hline$\hat{0}$ & $103,98 \pm 5,19$ & $13,74 \pm 1,09$ & $13,63 \pm 0,97$ & $20,47 \pm 1,12$ & $20,48 \pm 0,71$ & $27,78 \pm 1,79$ \\
& $98,05 \pm 3,80$ & $13,08 \pm 1,21$ & $12,08 \pm 1,23$ & $20,46 \pm 2,04$ & $18,55 \pm 1,82$ & $26,96 \pm 2,16$ \\
$p$-valor & 0,0142 & 0,2611 & 0,0170 & 0,9980 & 0,0044 & 0,4587 \\
\hline
\end{tabular}

Quando se comparou o FMBV de animais do sexo masculino $(13,63 \pm 0,97 \mathrm{~mm})$ com os do sexo feminino $(12,08 \pm 1,23 \mathrm{~mm})$ foi verificada diferença significativa $(\mathrm{p}=0,0170)$.

A distância média entre o forame mandibular e o processo condilar (FMCOND) foi 19,06 $\pm 1,80 \mathrm{~mm}$, não havendo diferença entre as hemimandíbulas esquerdas $(19,14 \pm 1,99 \mathrm{~mm})$ e direitas $(18,99 \pm 1,72 \mathrm{~mm})$. Uma diferença significativa ao nível de $5 \%(\mathrm{p}=0,0044)$ foi encontrada quando foi comparado o FMCOND de mandíbulas de animais dos sexos masculino $(20,48 \pm$ $0,71 \mathrm{~mm})$ e feminino $(18,55 \pm 1,82 \mathrm{~mm})$.

A distância média entre o forame mandibular e o processo angular (FMANG) foi 20,46 $\pm 1,82 \mathrm{~mm}$, não sendo verificada diferença entre os antímeros direito $(20,19 \pm 2,35 \mathrm{~mm})$ e esquerdo $(20,71 \pm 1,23 \mathrm{~mm})$ nem entre os sexos masculino $(20,47 \pm 1,12 \mathrm{~mm})$ e feminino $(20,46 \pm 2,04 \mathrm{~mm})$.

A distância média entre o forame mandibular e o processo coronoide (FMCOR) foi 27,17 $\pm 2,05$ mm, não havendo diferença significativa entre antímeros esquerdo $(27,42 \pm 2,11 \mathrm{~mm})$ e direito $(26,96 \pm 2,09 \mathrm{~mm})$ nem entre os sexos masculino $(27,78 \pm 1,79 \mathrm{~mm})$ e feminino $(26,96 \pm 2,16 \mathrm{~mm})$.

\section{Discussão}

A confirmação da existência de um canal mandibular com características semelhantes a dos demais mamíferos pressupõe que um bloqueio anestésico ao nível do forame mandibular possa ser efetuado também no cachorro-do-mato. As diferenças de formas observadas na comparação entre mandíbulas de Canis lupus familiaris (cão doméstico) e Cerdocyon thous (cachorrodo-mato) de portes semelhantes podem ser explicadas pelo fato de as superfícies ósseas proporcionalmente maiores do cão doméstico sirvam para inserção mais ampla da musculatura mastigatória, conferindo-lhe maior força de mordedura. De fato, a análise do conteúdo digestório do cachorro-do-mato revela predomínio de alimentos que não requerem mordedura potente como plantas, frutos, insetos e pequenos vertebrados (roedores, pássaros e anuros), diferente da alimentação do gênero Canis (LANGGUTH, 1975; BERTA, 1982). Entretanto, a diferença mais relevante com vistas ao bloqueio anestésico do nervo alveolar inferior é a posição relativamente mais rostral do forame mandibular no cachorro-do-mato. Isto sugere que a extrapolação de pontos de referências anatômicas, ainda que entre espécies de canídeos, pode não produzir o mesmo efeito.

As medidas ELM e ETM não têm relação direta com o forame mandibular, mas conferem uma ideia do comprimento longitudinal e largura do corpo da mandíbula ao nível da transição entre os dentes prémolares e molares inferiores, respectivamente. Com relação às demais quatro medidas que tomavam o forame mandibular como referência, também não houve diferença entre antímeros. No entanto, as medidas FMBV e FMCOND se mostraram significativamente maiores em indivíduos do sexo masculino. Diferenças no tamanho da cabeça entre machos e fêmeas podem explicar estes achados. Tais diferenças foram apontadas por Machado e Hingst-Zaher (2009) que identificaram importante dimorfismo sexual e geográfico por meio de avaliação craniométrica do Cerdocyon thous, ainda que as mandíbulas não tenham sido aferidas. Barroso et al. (2009) também indicaram variação na distância entre o forame mandibular e a borda ventral da mandíbula em felinos determinada, todavia, entre diferentes antímeros e não entre sexos. Diferenças significativas entre antímeros também foi verificada no posicionamento do 
forame mandibular em seres humanos por Strini et al. (2006).

De fato, o dimorfismo sexual é uma importante fonte de variabilidade intraespecífica e, deste modo, estudos em anatomia aplicada devem sempre considerar diferenças entre machos e fêmeas (TAKEMURA et al., 2003). Nossa avaliação recomenda que o dimorfismo sexual deva ser observado durante a execução da técnica do bloqueio local do nervo alveolar inferior no cachorrodo-mato e permite especular que diferenças na topografia do forame possam existir também entre antímeros ou sexos em espécies ainda não estudadas.

Ainda que procedimentos de bloqueios anestésicos do nervo alveolar inferior sejam considerados frequentes, de fácil execução e exijam emprego de referências anatômicas (GROSS et al., 1997; HOLMSTROM et al, 2004; HALE, 2007; EGGER; LOVE, 2009), notase uma escassez de estudos morfométricos do forame mandibular em animais. Barroso et al. (2009) publicaram dados referentes à morfometria deste forame aplicada à anestesia em felinos domésticos, mas não existem análises com esta ênfase em canídeos domésticos ou silvestres. Villamizar et al. (2009) estudaram o trajeto do canal mandibular em cães braquicéfalos das raças Boxer, Bulldog e Pug por meio de tomografia computadorizada e, entre outras medidas, determinaram que a distância entre o forame mandibular e a borda ventral da mandíbula é 10,10 $\pm 1,79 \mathrm{~mm}$. Com a mesma metodologia, Villamizar et al. (2010), posteriormente, demonstraram que esta distância em mandíbulas de cães mesaticéfalos é 9,09 $\pm 1,42 \mathrm{~mm}$. No cachorro-do-mato, que tem o crânio mesaticéfalo, esta distância foi 12,54 $\pm 1,39 \mathrm{~mm}$, sugerindo novamente que o emprego de resultados observados em cães domésticos possa não ser o mais adequado em canídeos silvestres.

Os principais pontos de referências palpáveis para a técnica do bloqueio anestésico local do nervo alveolar inferior são o processo angular e a borda ventral da mandíbula. Com base nas mensurações FMBV e FMANG, pode-se recomendar que a agulha devesse ser introduzida obliquamente $20,4 \mathrm{~mm}$ a partir do processo angular e perpendicularmente 12,1 mm (fêmeas) ou 13,6 $\mathrm{mm}$ (machos) a partir da borda ventral da mandíbula para que o anestésico seja administrado próximo ao forame mandibular e, deste modo, haja maior chance de causar o bloqueio pretendido.

Dados sobre a localização anatômica precisa do forame mandibular prestam contribuição na elaboração de técnicas eficientes de bloqueio para procedimentos dentários nas diferentes espécies e/ou raças. Isto é importante, pois a sanidade da cavidade oral está diretamente relacionada à manutenção de um bom estado nutricional, aumento da capacidade reprodutiva, da longevidade e da qualidade de vida dos carnívoros silvestres em cativeiro (PACHALY; GIOSO, 2001).

\section{Agradecimentos}

Ao biólogo Guilherme Andreoli (coordenador do projeto Fauna Viva) e à Concessionária Rio-Teresópolis (CRT) pela cessão dos cadáveres para estudo.

\section{Referências}

BARROSO, R. M. V.; FERREIRA, F. A.; SILVA, R. M.; LIMA, E. M. M. Morphometric analysis of the mandibular foramen of cats (Felis catus, Linnaeus 1758) with no defined breed. Bioscience Journal, Uberlândia, v. 25, n. 4, p. 135-142, 2009.

BECKMAN, B.; LEGENDRE, L. Regional Nerve Blocks for Oral Surgery in Companion Animals. Compendium: Small Animal/ Exotics, Yardley, v. 24, n. 6, p. 439-444, 2002.

BERTA, A. Cerdocyon thous. Mammalian species, Washington, $\mathrm{n}$. 186, p. 1-4, 1982.

CHEIDA, C. C.; NAKANO-OLIVEIRA, E.; FUSKO-COSTA, R.; ROCHA-MENDES, F.; QUADROS, J. Ordem Carnívora. In: REIS, N. R.; PERACCHI, A. L.; PEDRO, W. A.; LIMA, I. (Ed.). Mamíferos do Brasil. 2. ed. Londrina: Nelio R. dos Reis, 2011. p. 235-288.

DELlMANN, H. D.; MCCLURE, R. C. Sistema Nervoso do Carnívoro. In: GETTY, R. (Ed.). Anatomia dos Animais Domésticos. v. 2. 5 ed. Rio de Janeiro: Guanabara Koogan, 1986. p. 1569-1634.

DYCE, K. M.; SACK, W. O.; WENSING, C. J. G. Tratado de Anatomia Veterinária. 3. ed. Rio de Janeiro: Elsevier, 2004. 813 p.

EGGER, C.; LOVE, L. Local and regional anesthesia techniques, Part 3: Blocking the maxillary and mandibular nerves. Veterinary Medicine, Lenexa, v. 104, n.6, p. 288-291. 2009.

EVANS, H. E.; KITCHELL, R. L. Cranial nerves and cutaneous innervation of the head. In: EVANS, H. E. (Ed.). Miller's Anatomy of the Dog. 3. ed. Philadelphia: Saunders, 1993. p. 953-987.

EVANS, H. E.; LAHUNTA, A. Guide to the dissection of the dog. 7. ed. Missouri: Saunders, 2010. 303 p. 
GROSS, M. E.; POPE, E. R.; OBRIEN, D.; DODAM J. R.; POLKOW-HAIGHT, J. Regional anesthesia of the infraorbital and inferior alveolar nerves during noninvasive tooth-pulp stimulation in halothane-anesthetized dogs. Journal of the American Veterinary Medical Association, Schaumburg, v. 211, n. 11, p. 1403-1405, 1997.

HALE, F. Local anesthesia in veterinary dentistry. The CUSP. July, 2007. Disponível em: <http://www.toothvet.ca/PDFfiles/ LocalAnesthesia.pdf> . Acesso em: 14 ago. 2012.

HOLMSTROM, S. E.; FITCH, P. E.; EISNER, E. R. Veterinary Dental Techniques for the Small Animal Practitioner. 3. ed. Philadelphia: Saunders, 2004. 689 p.

INTERNATIONAL COMMITTEE ON VETERINARY GROSS ANATOMICAL NOMENCLATURE. Nomina Anatomica Veterinaria. 5. ed. Knoxville: Editorial Committee, 2012. 177 p.

KIERDORF, U. How many mandibles in a mammal? A note on terminology. Anatomia, Histologia, Embryologia, Berlin, v. 40, n. 2, p. 107-111, 2011.

KÖNIG, H. E.; LIEBICH, H.; CERVENY, C. Sistema Nervoso. In: KÖNIG, H. E.; LIEBICH, H. (Ed.). Anatomia dos Animais Domésticos: texto e atlas colorido. 4 ed. Porto Alegre: Artmed, 2011. p. 509-580.

LANGGUTH, A. Ecology and Evolution in the South American Canids. In: FOX, M.W. (Ed.) The wild Canids: their systematics, behavioral ecology and evolution. Washington: Dogwise Publishing, 1975. p. 192-206.

LIMA, D. S. C.; FIGUERÊDO, A. A.; ROCHA, E. A.; COSTA, V. H. M. V.; CASTRO, M. P.; SILVA, R. C. P.; CHAGAS, G. L.; ARAÚJO, L. P.; MENDONÇA, V. R. R.; GRAVINA, P. R.; MENESES, J. V. L. Estudo anatômico do forame mandibular e suas relações com pontos de referência do ramo da mandíbula. Revista Brasileira de Cirurgia Craniomaxilofacial, São Paulo, v. 14, n. 2, p. 91-96, 2011.

MACHADO, F. A.; HINGST-ZAHER, E. Investigating South American biogeographic history using patterns of skull shape variation on Cerdocyon thous (Mammalia: Canidae). Biological Journal of the Linnean Society, London, v. 98, p. 77-84, 2009.
PACHALY, J. R. GIOSO, M. A. The oral cavity. In: FOWLER, M. E.; CUBAS, Z. S. (Ed.). Biology, medicine and surgery of South American wild animals. Ames: Iowa State University Press, 2001. p. 457-463.

PESSUTTI, C.; SANTIAGO, M. E. B.; OLIVEIRA, L. T. F. Order Carnivora Family Canidae (Dogs, foxes and maned wolves). In: FOWLER, M. E.; CUBAS, Z. S. (Ed.). Biology, medicine and surgery of South American wild animals. Ames: Iowa State University Press, 2001. p. 279-290.

RIZZOLO, R. J. C.; MADEIRA, M. C. Anatomia facial com fundamentos de anatomia sistêmica geral. 2. ed. São Paulo: Sarvier, 2006. 343 p.

STRINI, P. J. S. A.; SILVA-JÚNIOR W.; RODRIGUES, D. A.; STRINI, P. J. S. A.; GUIMARÃES, E. C.; BERNARDINOJÚNIOR, R. Avaliação topográfica do forame mandibular em peças anatômicas maceradas parcialmente dentadas e edêntulas. Revista da Faculdade de Odontologia da Universidade de Passo Fundo, Passo Fundo, v. 11, n. 2, p. 111-115, 2006.

TAKEMURA, C. M.; CESAR-JÚNIOR, R. M.; HINGST-ZAHER, E.; ARANTES, R. A. T.; COSTA, L. F. Application of wavelets in the analysis of sexualdimorphism in the skull of South American canids (Mammalia, Canidae). 2003. Disponível em: <http://butantan.academia.edu/ErikaHingstZaher/ Papers/936066/Application_of_wavelets_in_the_analysis_of sexual_dimorphism_in_the_skull_of_South_American_canids_ Mammalia_Canidae_>. Acesso em: 12 ago. 2012.

VILLAMIZAR, L. A.; GIOSO, M. A.; LOBOS, C. M. V.; PINTO, A. C. B. C. F. Localization of the mandibular canal in brachicephalic dogs using computed tomography. Journal of Veterinary Dentistry, Gaithersburg, v. 26, n. 3, p. 156-163, 2009.

VILLAMIZAR, L. A.; GIOSO, M. A.; LOBOS, C. M. V.; PINTO, A. C. B. C. F. Determinação do trajeto do canal mandibular por meio de tomografia computadorizada em dez mandíbulas de cadáveres de cães mesaticefálicos. Brazilian Journal of Veterinary Research and Animal Science, São Paulo, v. 47, n. 4, p. 274-281, 2010. 